\title{
conomics
}

The Open-Access, Open-Assessment E-Journal

Vol. 3, 2009-22| June 8, 2009 | http://www.economics-ejournal.org/economics/journalarticles/2009-22

\section{Superstatistics of Labour Productivity in Manufacturing and Nonmanufacturing Sectors}

\author{
Hideaki Aoyama \\ Department of Physics, Kyoto University, Kyoto \\ Yoshi Fujiwara \\ ATR Laboratories, Kyoto \\ Yuichi Ikeda \\ Hitachi Ltd, Hitachi Research Laboratory, Ibaraki \\ Hiroshi Iyetomi \\ Department of Physics, Niigata University, Niigata \\ Wataru Souma \\ College of Science and Technology, Nihon University, Chiba
}

\begin{abstract}
Labour productivity distribution (dispersion) is studied within the framework of statistical physics and the result is compared with the outcome of the empirical analysis. Superstatistics is presented as a natural theoretical framework for the productivity distribution. The demand index $\kappa$ is proposed within this framework as a new business index. Productivity analysis is made on the Japanese data covering smallto-medium to large firms from 1996 to 2006 and the power-law for both firms and workers is established. The demand index $\kappa$ is evaluated in the manufacturing sector. A new discovery is reported for the nonmanufacturing (service) sector, which calls for an expansion of the superstatistics framework to include the negative temperature range.
\end{abstract}

Special issue Reconstructing Macroeconomics

JEL: E10, E30, O40

Keywords: Labour productivity; superstatistics; Pareto’s Law; business cycle; demand index

\section{Correspondence}

Hideaki Aoyama, Physics Department, Kyoto University, Kyoto 606-8501, Japan;

e-mail: hideaki.aoyama@scphys.kyoto-u.ac.jp

The authors would like to thank Professor Hiroshi Yoshikawa for previous collaborative work and Professor Masanao Aoki for encouraging them at various stages of their research in econophysics over the years. They would also like to thank the CRD association and its chairman, Mr. Shigeru Hikuma, for his help and advice in using their database. Computing Facility at the Yukawa Institute for Theoretical Physics was used for part of the numerical computation. 


\section{Introduction}

Standard equilibrium theory in economics implies that labour productivity is equal among firms and sectors. This was shown to be wrong by a detailed study of the real data (Aoyama et al., 2008). Facing this situation, one may argue that (i) since the real data is slightly different from ideal cases that the economic theory deals with and is contaminated with various inaccuracies and errors, a slight deviation from the theoretical prediction is unavoidable and is even expected. Moreover, (ii) since the equilibrium theory is self-consistent, reasonable and convincing, it must be true. These claims are not valid, as (i) the distribution of productivity is wide-spread; it is not even a normal distribution or log-normal distribution as expected from the contamination argument, but does obey Pareto's Law (the power law) (Pareto, 1896) for large productivity, that is, the distribution has the distinct characteristics of fat tails, and (ii) there may exist other theories that are far more convincing and the validity of the theory can be judged only by facing the true nature of the subject. Indeed, physics, or any other discipline of exact science managed to develop to the current status just by following (ii): No matter how much the pre-Copernican theory is reasonable, beautiful and convincing, the earth still moves; no matter how the idea of absolute time in Newtonian mechanics (which underlies the current equilibrium theory of economics) irrefutable, Einstein's relativity theory describes the true nature of time and space. These and numerous other historical examples in exact science teach us that we need to investigate the phenomena vigorously and must construct theories that meets the demands of true science. Simply put, we need to take a scientific approach.

Such was the thought behind the study of productivity by Aoyama et al. (2008), who proposed the superstatistics theory in statistical physics as a theoretical framework for labour productivity.

In this paper, we further advance the superstatistics theory of productivity by examining the whole spectrum of firms in Japan, while in the previous work of Aoyama et al. (2008) and Aoyama et al. (2009), the data was limited to listed firms. Furthermore, we analyse the manufacturing and nonmanufacturing sectors separately. In Section II (and in the Appendix), we present the superstatistics framework for productivity for completeness. Then in Section III, after explaining the nature of the database and the method of analysis, we present the results for the manufacturing sector and the nonmanufacturing sector separately. We also study the distribution of productivity of business sectors. Section IV contains some conclusions and discussions on the necessity of expanding the superstatistics framework.

\section{Superstatistics Theory of Productivity}

We first review the superstatistics theoretical framework of productivity proposed by Aoyama et al. $(2008,2009)$ in a concise manner. Data analysis and a discussion of the evaluation of the property of the aggregate demand are done in later sections using this framework. 


\section{$2.1 \quad$ Statistics}

Yoshikawa and Aoki (Yoshikawa, 2003, Aoki and Yoshikawa, 2007) proposed an equilibrium theory of productivity distribution several years ago. Its essence is the equilibrium theory of statistical physics, where the most common distribution is realized under given constraints. Its beauty lies in the fact that it does not depend on the details of individual properties and interactions among constituents (firms in economics and atoms and molecules in statistical physics). Let us first review it very briefly.

We label firms with the index $k=1,2, \ldots, K$, where $K$ is the total number of firms, the number of workers at the $k$-th firm by $n_{k}$, productivity of the $k$-th firm by $c_{k}$, all for a given year. There are two constraints on these quantities.

\section{(i) Total number of workers $N$ :}

$$
\sum_{k=1}^{K} n_{k}=N .
$$

(ii) The aggregate demand $\tilde{D}$ : The sum of a firm's production is the total production, which is equal to the aggregate demand $\tilde{D}$;

$$
\sum_{k=1}^{K} n_{k} c_{k}=\tilde{D}
$$

Implicit here is that we are dealing with the mean labour productivity

$$
c:=\frac{Y}{L}
$$

where $Y$ is the value added and $L$ is the labour (in number of workers). Although this is different from the marginal labour productivity $c^{\text {(marginal) }}:=$ $\partial Y / \partial L$ relevant in standard equilibrium theory, this difference is irrelevant as we will elaborate later.

By using the standard proposition that the distribution that maximizes the probability under these constraints is realized in nature, which is equivalent to the entropy-maximization, the Boltzmann law is obtained. This states that the probability $p_{k}$ of the worker's productivity being equal to $c_{k}$ is the following.

$$
p_{k}:=\frac{\left\langle n_{k}\right\rangle}{N}=\frac{1}{Z(\beta)} e^{-\beta c_{k}},
$$

where $Z(\beta)$ is the usual partition function:

$$
Z(\beta):=\sum_{k=1}^{K} e^{-\beta c_{k}} .
$$


This guarantees the normalization of the probability $p_{k}$;

$$
\sum_{k=1}^{K} p_{k}=1 .
$$

The parameter $\beta$ is inverse-temperature determined by the mean demand $D$ as follows:

$$
D:=\frac{\tilde{D}}{N}=-\frac{d}{d \beta} \ln Z(\beta) .
$$

In our database, we have nearly half a million firms and about 10 million workers. Therefore, it is most appropriate to use the continuous notation, in which the probability distribution function (pdf) of the firm's productivity is denoted by $p^{(\mathrm{F})}(c)$, and the pdf of the worker's productivity by $p^{(\mathrm{W})}(c)$. From Eq.(4), they satisfy the following:

$$
p^{(\mathrm{W})}(c)=\frac{1}{Z(\beta)} e^{-\beta c} p^{(\mathrm{F})}(c)
$$

where the partition function is

$$
Z(\beta):=\int_{o}^{\infty} e^{-\beta c} p^{(\mathrm{F})}(c) d c
$$

\subsection{Superstatistics}

Although the theoretical prediction (8) is both elegant and powerful, it is quite limited in the sense that it is realized in a stationary environment, namely, that demand (and thus temperature) has to be constant. However, the demand is rarely constant. Rather it is one of the most quickly changing parameters (Yoshikawa, 2003). Therefore, we need to expand the horizon of the theory to meet the changing environment. Just such a theory, named superstatistics (the statistics of statistics), has been proposed recently by Beck and Cohen (2003) in the context of statistical physics. In this theory, the system goes through changing external influences, but is in equilibrium described by the Boltzmann distribution (8) within a certain limited scale in time and/or space. Therefore, the whole system can be described by an average over the Boltzmann factors, with the weight given by the relative scales (in time and space) of the temperature $(1 / \beta)$, which the system experiences.

This superstatistics was successfully applied to various systems (Beck, 2005, 2008). Most analogous to our economic system of firms and workers may be the Brownian motion of a particle going thorough changing temperature and viscosity (Ausloos and Lambiotte, 2006, Luczka and Zaborek, 2004). Our workers are the particles in Brownian motion: They move from firm to firm, each of which keeps trying to meet ever-changing demand by employing and dismissing workers. Therefore, a superstatistics is the right framework to deal with the distribution of the workers. 
A weighted average over the temperature now replaces the Boltzmann factor $e^{-\beta c}$ :

$$
B(c)=\int_{0}^{\infty} e^{-\beta c} f_{\beta}(\beta) d \beta .
$$

In this equation the changing environment is represented by the weight factor $f_{\beta}(\beta)$, which is, in turn, a function of the mean demand $D$ by Eq.(7). The pdf of workers' productivity (8) is now modified to;

$$
p^{(\mathrm{W})}(c)=\frac{1}{Z_{B}} p^{(\mathrm{F})}(c) B(c) .
$$

The new partition function $Z_{B}$ in the above is given by,

$$
Z_{B}=\int_{0}^{\infty} p^{(\mathrm{F})}(c) B(c) d c
$$

Let us study what the superstatistics theory tells us for the high productivity region. We concentrate on this region because, as we will see by the data analysis in the next section, both the firm's productivity and worker's productivity obeys Pareto's Law (the power law):

$$
\begin{aligned}
p^{(\mathrm{F})}(c) & \propto c^{-\mu_{\mathrm{F}}-1}, \\
p^{(\mathrm{W})}(c) & \propto c^{-\mu_{\mathrm{W}}-1} .
\end{aligned}
$$

This feature brings advantages to the study of high-productivity because of the following reasons:

(i) It is quite evident in the data and the Pareto indices $\mu_{\mathrm{F}, \mathrm{W}}$ can be estimated reliably. In comparison, the medium-to-low range sometimes shows a two-peak structure, which makes it difficult to extract notable, representative features. (Elsewhere in this volume, Souma et al. (2009) elaborates on this point.)

(ii) As was proven by Aoyama et al. (2008), if Pareto's Law holds for "mean" productivity $c$, the same law with the same value of the Pareto index holds for marginal productivity $c^{\text {(marginal) }}$ under a wide assumption.

Let us now study the behaviour of Eq.(10) for large $c$. This integration is dominated by the small $\beta$ region. Thus the behaviour of the $\operatorname{pdf} f_{\beta}(\beta)$ for small $\beta$ is critical. Let us assume the following in this range:

$$
f_{\beta}(\beta) \propto \beta^{-\gamma} \quad(\gamma<1)
$$

where the constraint for the parameter $\gamma$ comes from the convergence of the integration in Eq.(10). This leads to the following for large $c$ :

$$
B(c) \propto \Gamma(1-\gamma) c^{\gamma-1}
$$

Substituting this and the Pareto laws Eqs. (13) and (14) into Eq. (11), we obtain 
the following:

$$
\mu_{\mathrm{W}}=\mu_{\mathrm{F}}-\gamma+1
$$

We note here that because of the constraint $\gamma<1$, this leads to the inequality

$$
\mu_{\mathrm{W}}>\mu_{\mathrm{F}}
$$

This becomes a critical test of the superstatistics theory of productivity, which we come back to in the following section.

The above derivation of Eq.(17) proves, in effect, that the Pareto law for firms and that for workers are compatible only if the temperature distribution obeys Eq. (15), as no other behaviour could result in the power law (16). In this sense, we see that empirical observation leads to Eq. (fbeta). This in turn leads to empirical laws to the distribution and fluctuation of the demand through Eq. (7): In this manner, the parameter $\gamma$ in the distribution of $\beta$ is related to a parameter in the distribution of $D$, which we denote by $f_{D}(D)$ by the following relation:

$$
f_{\beta}(\beta) d \beta=f_{D}(D) d D
$$

The mathematical relation between $\beta$ and $D$ is studied in detail in the appendix. Using the result (44) and Eq.(15), we find that

$$
f_{D}(D) \propto\left(\langle c\rangle_{0}-D\right)^{-\delta}
$$

with

$$
\gamma-1= \begin{cases}\delta-1 & \text { for } 2<\mu_{\mathrm{F}} \\ \left(\mu_{\mathrm{F}}-1\right)(\delta-1) & \text { for } 1<\mu_{\mathrm{F}}<2 .\end{cases}
$$

Note that $f_{D}(D)$ has an upper limit $\langle c\rangle_{0}$ : From Eq.(8), it is evident that as the temperature $1 / \beta$ goes up, workers move to firms with higher productivity. As the temperature reaches infinity $\beta=0$, all the firms have the same number of workers. Thus the total demand is limited by the values achieved at this point, where $D=$ $\langle c\rangle_{0}$.

Combining Eqs.(17) and (21), we reach the following relation between the Pareto indices:

$$
\mu_{\mathrm{W}}= \begin{cases}\mu_{\mathrm{F}}-\delta+1 & \text { for } 2<\mu_{\mathrm{F}} \\ \left(\mu_{\mathrm{F}}-1\right)(-\delta+1)+\mu_{\mathrm{F}} & \text { for } 1<\mu_{\mathrm{F}}<2 .\end{cases}
$$

This relation between $\mu_{W}$ and $\mu_{F}$ is illustrated in Fig.1. The range of the parameter $\delta$ is $-\infty<\delta<1$ from the normalizability of the distribution of $f_{D}(D)$. The upper limit may also be obtained from the constraint $\gamma<1$ and Eq.(21). Because of this, Eq.(22) predicts that $\mu_{\mathrm{W}}$ is larger than $\mu_{\mathrm{F}}$. Also, Eq.(22) has a fixed point at $\left(\mu_{\mathrm{W}}, \mu_{\mathrm{F}}\right)=(1,1)$; the line defined by Eq.(22) always passes through this point irrespective of the value of $\delta$. The Pareto index for firms is smaller than that for workers, but it cannot be less than one, because of the existence of this fixed point. 


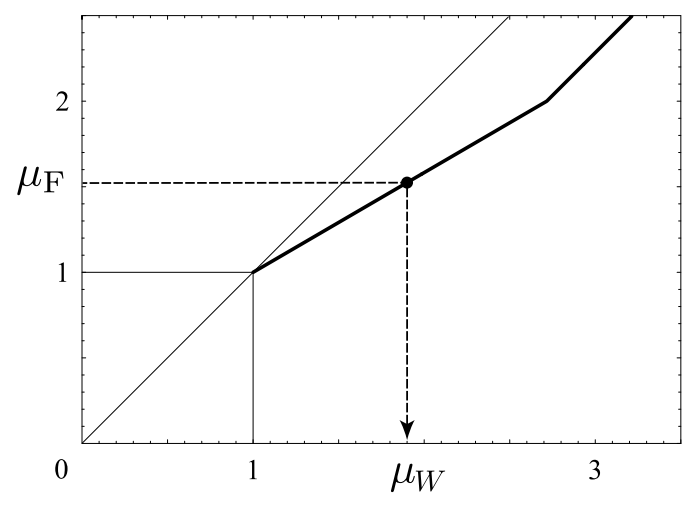

Figure 1: An illustration of the relation between $\mu_{\mathrm{W}}$ and $\mu_{\mathrm{F}}(22)$. The solid line is the relation $(22)$, and the filled circle is the data.

In this way, the parameter $\delta$, calculated from $\mu_{\mathrm{F}}$ and $\mu_{\mathrm{W}}$, represents the behaviour of the demand close to its upper limit. As a parameter with the same function, we propose the following parameter, which we call Demand Index:

$$
\kappa:=\frac{1}{2-\delta}
$$

This parameter is a monotonically increasing function of $\delta$ and ranges from 0 to 1 . The limited range of $\kappa$ make it easy to handle and plot. If $\kappa$ is close to one, the demand fluctuates to the high region significantly; if it is equal to zero, the demand does not go very high (it could be dumping faster than any power law toward the upper limit).

More generally, a function $(A-1) /(A-\delta)$ with $A>1$ has the required property, but by choosing $A=2$ we obtain $d \kappa /\left.d \delta\right|_{\delta=1}=1$, so that $\kappa \simeq \delta$ for $\delta \rightarrow 1$. This proximity of $\kappa$ and $\delta$ is desirable to some extent as the data often shows $\delta$ in the range 0.5 to 1 .

In summary, the superstatistics framework predicts that the Pareto indices $\mu_{\mathrm{W}}$ and $\mu_{\mathrm{F}}$ determine the Demand Index $\kappa$ as follows:

$$
\kappa= \begin{cases}\frac{1}{\mu_{\mathrm{W}}-\mu_{\mathrm{F}}+1} & \text { for } 2<\mu_{\mathrm{F}} \\ \frac{\mu_{\mathrm{F}}-1}{\mu_{\mathrm{W}}-1} & \text { for } 1<\mu_{\mathrm{F}}<2 .\end{cases}
$$

This relation is illustrated in Fig.2.

\section{Empirical Facts}

\subsection{Database and the Analysis}

In calculating the productivity $c$ by Eq.(3) from data, we calculate the value added $Y$ by the method put forward by the Bank of Japan (Souma et al., 2009), which is 


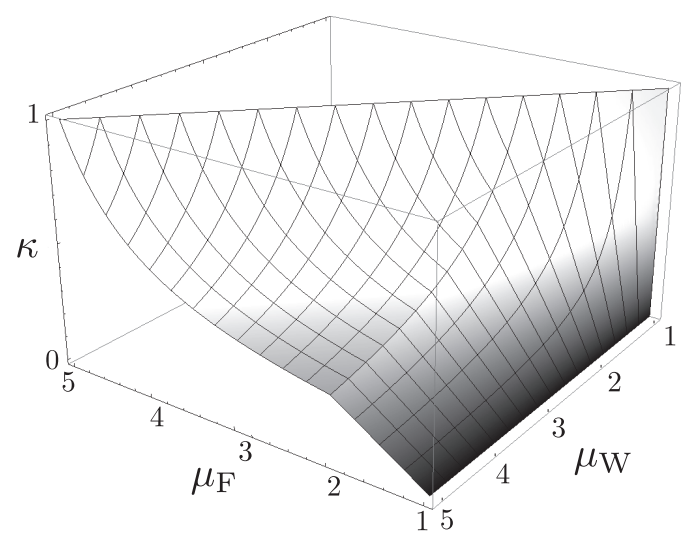

Figure 2: The demand index $\kappa$ as a function (24) of $\mu_{\mathrm{F}}$ and $\mu_{\mathrm{W}}$.

the most common method used in Japan. As for the number of workers $L$, we use the average of the value of that year and that of the past year, as each are defined to be the value at the end of the year.

For comprehensive, high-accuracy study, we constructed a database derived from the following two sources:

Nikkei-NEEDS Nikkei Economic Electronic Databank System (NEEDS) database is a commercial product available from Nikkei Media Marketing, Inc. (Nikkei Media Marketing, 2008) and contains financial data from all the listed firms in Japan. This is a well-established and representative database, widely used for various purposes from research to practical business applications. We have extracted data from their 2007 CD-ROM version, which contains two to three thousand firms and five to six million workers.

CRD Credit Risk Database (Credit Risc Database Association, 2008) is the first and only database for small-to-medium firms in Japan. It started collecting data from both banks and credit guarantee corporations (Credit Guarantee Corporations, 2008) since 2001. The latter, however, does not contain enough database entry necessary for the Bank-of-Japan method and thus are omitted from our database.

By combining these two databases and removing any overlap, we have obtained a unified database that covers a wide range of firms in Japan. ${ }^{1}$ The total number of firms and workers covered in this unified database is plotted in Fig.3.

Unfortunately, the CRD data covers only the period from 1996 to 2006, and does not go far enough into the past, unlike the Nikkei-NEEDS. This limits our unified database to the same short period. We, however, consider that it is important to include small-to-medium firms in our analysis so that we have a view of the whole spectrum of firms and workers in Japan, hence our decision to create and analyse this database. In coming years, we trust that the CRD will keep collecting data. So, we may extend our analysis to the future, if not the past, by continuing and expanding what we have started here in this paper.

\footnotetext{
${ }^{1}$ The same database was used for productivity analysis by Ikeda and Souma (2009).
} 

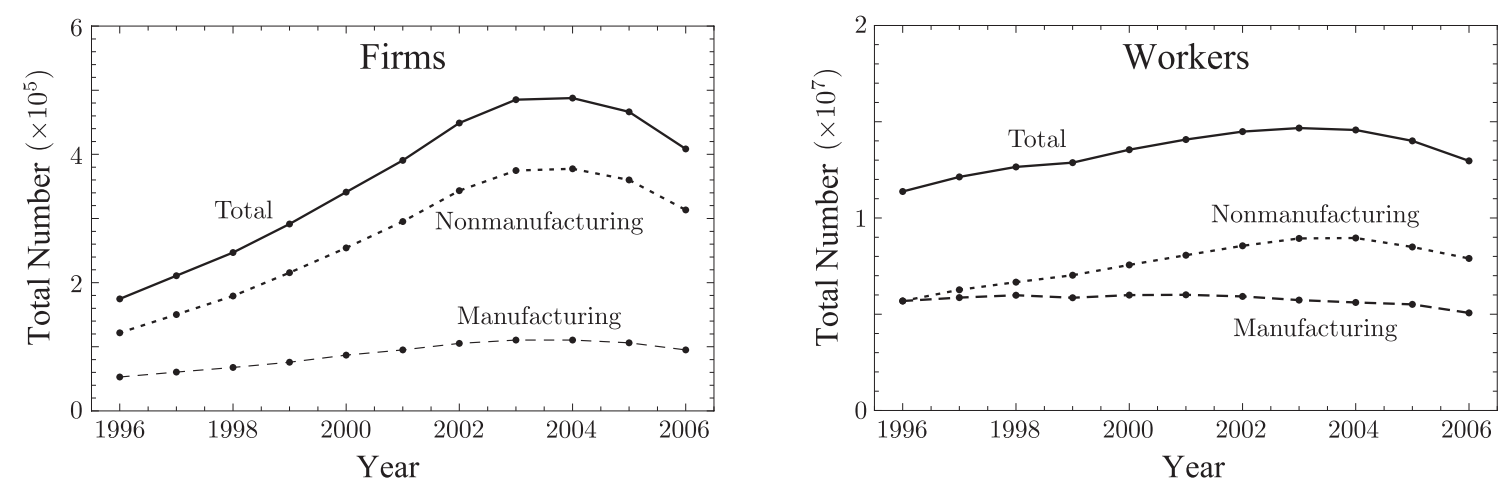

Figure 3: Total number of firms (left) and workers (right) in our database, both for the manufacturing sector (dashed lines), the nonmanufacturing sector (dotted lines) and both sectors (solid lines).

We need a model distribution to fit the data and extract the value of the Pareto indices. It has to have several properties: (i) It has be defined for the whole range, $c \in[0, \infty]$; (ii) it has to include the power law (13) and (14) for large $c$; and (iii) it has to be able to describe the data to some accuracy over the whole region. The power law manifests itself as a straight line in the log-log rank-size plots of the data (see Fig.4. ${ }^{2}$ ) Since its gradient is the Pareto index, its value is estimated by fitting the straight section of the rank-size plot with a straight line. Although this can be done easily and is also intuitive, it has several pitfalls: Often, the definition of "the straight section" is ad-hoc. A slight change can bring a significant change in the value of the Pareto index. Even if a good one can be found for a particular year, it might not work for other years of the same database, which makes a comparison of different years meaningless.

The "Generalized Beta Distribution of the Second Kind" (GB2) (Kleiber and Kotz, 2004) satisfies the property (i)-(iii) and yet, it is manageable. It is defined by the following pdf;

$$
p^{(\mathrm{GB} 2)}(c)=\frac{q}{B(\mu / q, \nu / q)} \frac{1}{c}\left(\frac{c}{c_{1}}\right)^{\nu}\left[1+\left(\frac{c}{c_{1}}\right)^{q}\right]^{-(\mu+\nu) / q},
$$

where the four parameters satisfy constraints $\mu, \nu, q, c_{1}>0$. Since for large $c$;

$$
p^{(\mathrm{GB} 2)}(c) \simeq\left(\frac{c}{c_{1}}\right)^{-\mu-1}
$$

the parameter $\mu$ is the Pareto index. Incidentally, its cumulative distribution functions (cdf) is the following:

$$
P_{>}^{(\mathrm{GB} 2)}(c):=\int_{c}^{\infty} p^{(\mathrm{GB} 2)}(c) d c .=\frac{B(z, \mu / q, \nu / q)}{B(\mu / q, \nu / q)},
$$

\footnotetext{
${ }^{2}$ Firms with an extremely high value of productivity are removed from this plot, as they often report just one worker, which, in view of their huge income, cannot be a reasonable representation of manpower.
} 

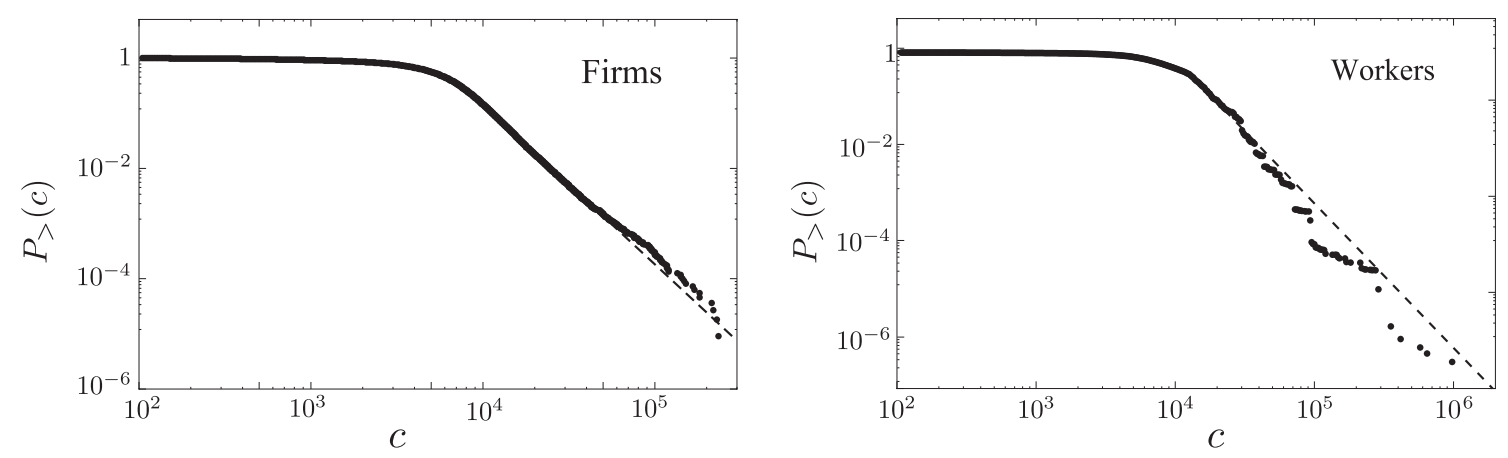

Figure 4: The rank-size plot of the productivity data (dots) and the best-fit cdf (dashed line) for firms (left) and workers (right) in 2004.

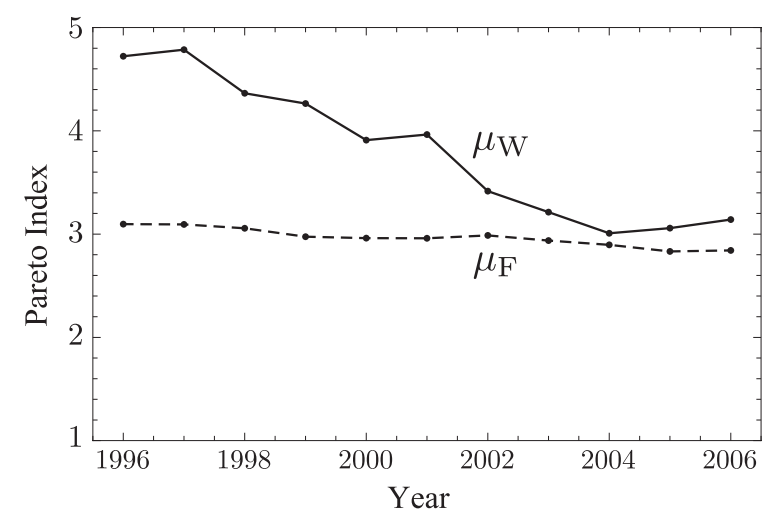

Figure 5: The Pareto indices $\mu_{\mathrm{F}}$ and $\mu_{\mathrm{W}}$ for the manufacturing sector.

where

$$
z=\left[1+\left(\frac{c}{c_{1}}\right)^{q}\right]^{-1},
$$

and $B(z, s, t)$ is the incomplete Beta function with $B(1, s, t)=B(s, t)$. (A detailed study of small-to-medium productivity was done by Souma et al. (2009) using this GB2 distribution.)

\subsection{Manufacturing Firms}

Rank-size plots of the productivity of the manufacturing sector in 2004 is given in Fig. 4 by dots, together with the best-fit cdf obtained by the maximum likelihood method. In these log-log plots, we see that the actual distributions of the data are close to straight lines for large $c$, which implies that it obeys the power law (i.e., the Pareto law) as we have discussed above. The best-fit cdf (dashed lines) indeed represents the data to good accuracy. The situation is also quite similar in all the other years.

The values of the Pareto indices $\mu_{\mathrm{F}}$ and $\mu_{\mathrm{W}}$ thus obtained are plotted in Fig.5. Substituting these values to Eq.(24), we obtained the value of the demand index $\kappa$ joined by solid lines in Fig.6. We see here that the demand is slowing rising during 


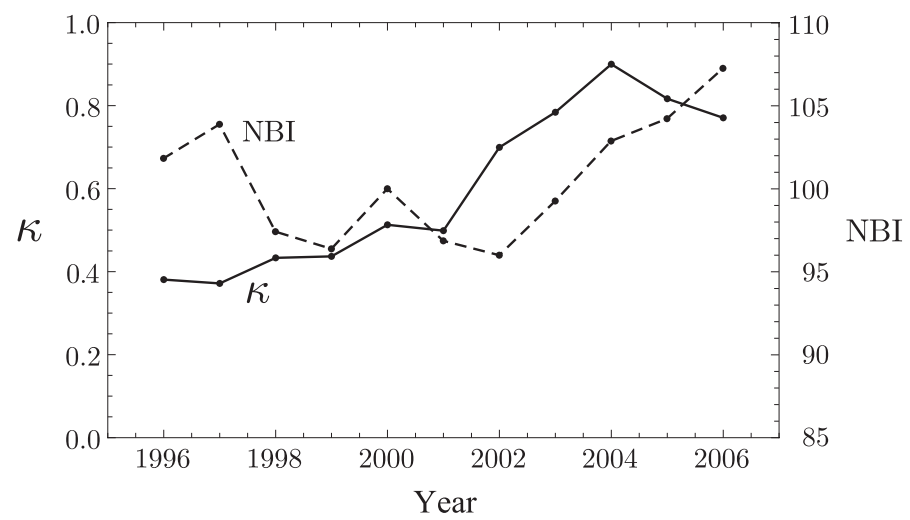

Figure 6: The demand index $\kappa$ for the manufacturing sector (solid lines) and the Nikkei Business Index (dashed lines).

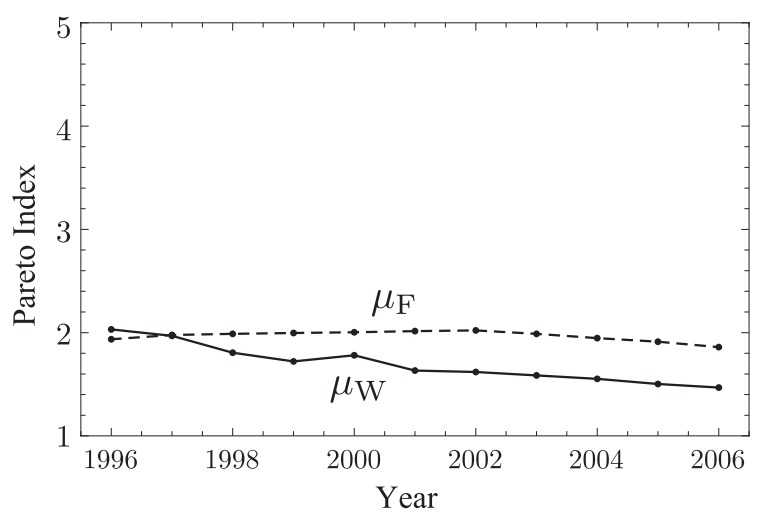

Figure 7: The Pareto indices $\mu_{\mathrm{F}}$ and $\mu_{\mathrm{W}}$ for the nonmanufacturing sector.

this period, which is in agreement with general observations in Japan. Plotted in Fig.6 with dashed lines is the Nikkei Business Index (NBI), which is a major business index in Japan (Nikkei Net Interactive, 2008). We observe here that their correlation is good to some extent, which is consistent with the fact that our demand index $\kappa$ provides a measure of demand.

\subsection{Nonmanufacturing (Service) Firms}

In the nonmanufacturing sector, the same analysis leads to the result plotted in Fig.7. It is quite notable that it is completely different from the manufacturing sector: The Pareto index $\mu_{\mathrm{F}}$ is larger than $\mu_{\mathrm{W}}$. Since the larger Pareto index means that the pdf is damped highly for large $c$, this means that the higher the productivity of a firm, the more workers are employed. This is not allowed under the ordinary Boltzmann distribution (8) due to the Boltzmann factor $e^{-\beta c}$. It is not allowed in the superstatistics either, since it is an weighted average over the Boltzmann distribution. Therefore, this behaviour of the nonmanufacturing sector calls for an expansion of the theoretical framework. 


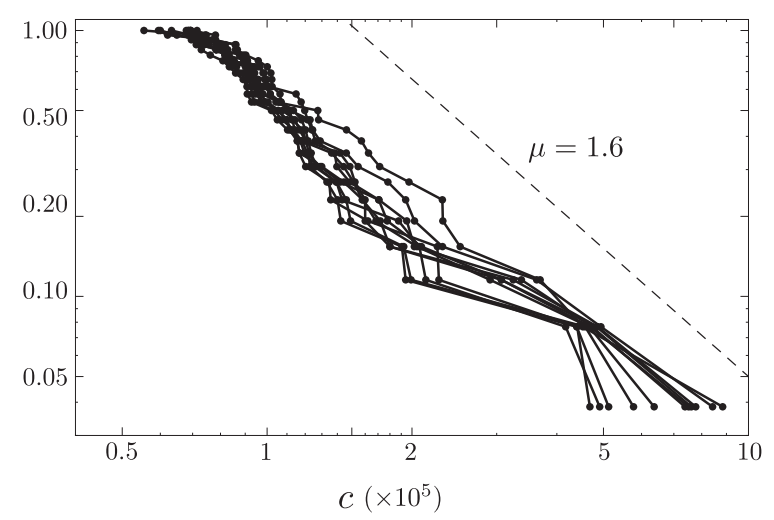

Figure 8: Productivity distribution of 26 sectors from 1996 to 2006. The dashed line is a power-law distribution with the Pareto index $\mu=1.6$.

\section{Productivity of Sectors}

Productivity of sectors are of interest. Our database, following Nikkei NEEDS, contains 26 sectors, among which 15 are manufacturing sectors and 12 nonmanufacturing sectors. Their productivity distributions from 1996 to 2006 is plotted in Fig.8. Evident in this plot is that the productivity distribution obeys the power-law with the Pareto index $\mu \simeq 1.6$ every year. Since the number of data is limited, unlike the firms and workers, fitting with GB2 distribution and estimating the value of $\mu$ are not very illuminating. In other words, the obtained values of $\mu$ would suffer from large statistical errors. It is more so if manufacturing sectors and nonmanufacturing sectors are studied separately.

The notable feature of the sector distribution is the (i) it is approximately Pareto and (ii) the values of the Pareto index are certainly lower than that of firms every year. This is in accordance with the idea of applying superstatistics framework to firms and sectors, in contrast to workers and firms (Aoyama et al., 2008): We may now think of a firm (instead of a worker) choosing a sector (instead of a firm) for its business activity. Applying the superstatistics to them, we find again that the Pareto index of the firms are higher than that of sectors. This is what we observe in Fig.8.

\section{Conclusion and Discussions}

We have studied the superstatistics theory of productivity and have introduced the demand index $\kappa$, which determines the relation between the Pareto indices of the productivity distributions of firms and workers. Analysis of the whole spectrum, from small to large, firms in Japan from 1996 to 2006 is carried out and the manufacturing sector was studied within the superstatistics framework.

One might argue that what we have been observing is a temporary situation and eventually, the effect of shocks, including the 1999 bubble collapse, would be averaged out. Moreover productivity would start converging to a unique value, as predicted by orthodox equilibrium theory. In order to study this point, we have examined changes in the productivity of sectors over the years. The results are 
(a)

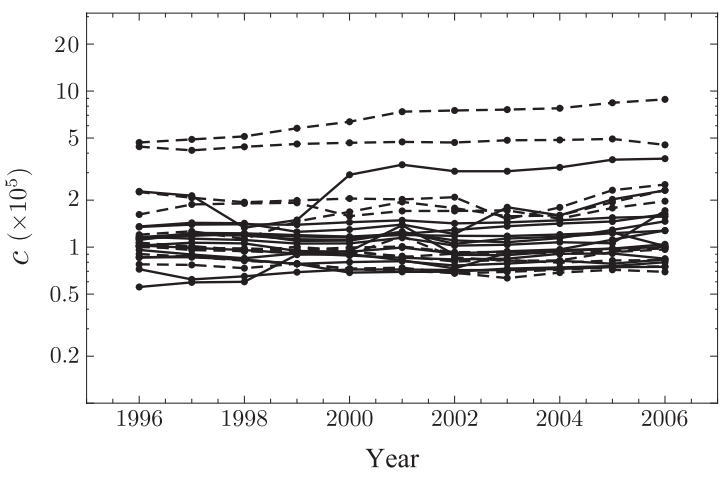

(b)

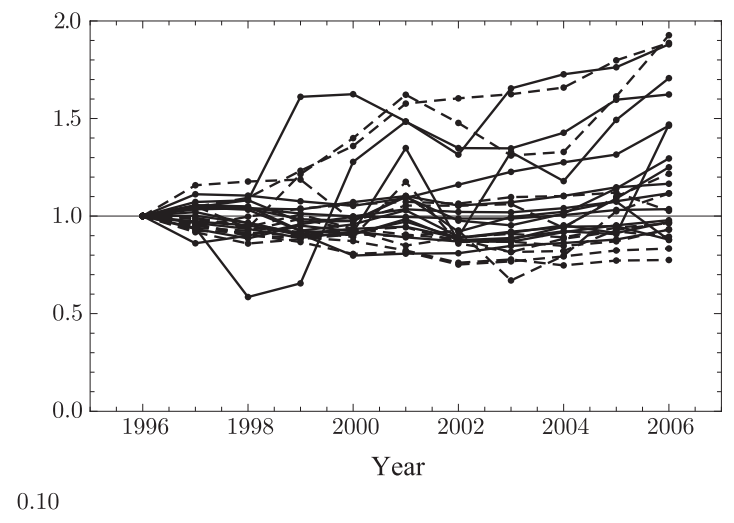

Figure 9: Evolution of productivity of each sector. (a) is the actual value, while (b) is the value normalized at 1996. Solid lines are of manufacturing sectors and dashed lines are of nonmanufacturing sectors.

plotted in Fig.9. Each line represents a sector, the solid lines for manufacturing and the dashed lines for nonmanufacturing. In this Figure, we see that they are far from arriving at a unique value. Rather, they keep fluctuating. Sometimes, a difference in productivity between sectors widens. This sort of behaviour is typical in physical systems. Distribution is maintained, while viewed in detail, just as each atom keeps changing its energy and momentum. Such is the nature of physical equilibrium and so are the economic systems.

We stress here that the distribution (20) of the demand $D$ is unique in the superstatistics framework: It has to obey this distribution if Pareto law hold for both firms and workers and Eq. (18) is satisfied. Just what kind of dynamics and economic principles underlies the demand distribution (20) is unknown. It would be quite interesting and important to construct a model which leads to this kind of behaviour. It is quite possible that such a model could be one of the building blocks useful and necessary for reconstructing macro-economics.

On the other hand, the nonmanufacturing (service) sector showed peculiar characteristics that have never been seen before. The Pareto index for worker was larger than that for firms.

In the ordinary Boltzmann distribution (10), the positivity of the temperature guarantees that the higher the productivity, the fewer the workers employed. Since superstatistics is the weighted average of the Boltzmann distribution, no matter what the weight function $f_{\beta}(\beta)$ is, the higher productively means fewer workers, which is the reason for $\mu_{\mathrm{F}}<\mu_{\mathrm{W}}$. The fact that the nonmanufacturing sector violates this constraint means that the weighted average over a negative temperature is required.

A negative temperature is possible for a physical system in nonequilibrium. One such example is a laser, where many atoms or electrons are in an excited state before the emission. For our current case, there is at least one economic reason why it is required; an excess of demand. There are certain limits to the productivity of a given firm due to the many constraints it faces. But by hiring more people while maintaining the same structure, firms can increase their value added, thereby 
meeting the increasing demand. Just such a situation could be happening in the nonmanufacturing industry.

The superstatistics of negative temperatures have not been developed yet: While it is easy to expand the weighted average over negative temperatures, the full consequences are not clear at this stage. It would therefore be quite interesting to develop this theory and use it to deal with the nonmanufacturing sector.

\section{Appendix: Temperature and Demand}

We first note the following three basic properties (i)-(iii).

(i) The temperature, $T=1 / \beta$ is a monotonically increasing function of the aggregate demand, $D$. We can prove it using Eq.(7) as follows:

$$
\frac{d D}{d T}=-\frac{1}{T^{2}} \frac{d D}{d \beta}=\beta^{2} \frac{d^{2}}{d \beta^{2}} \ln Z(\beta)=\beta^{2}\left(\left\langle c^{2}\right\rangle_{\beta}-\langle c\rangle_{\beta}^{2}\right) \geq 0,
$$

where $\left\langle c^{n}\right\rangle_{\beta}$ is the $n$-th moment of productivity defined as follows:

$$
\left\langle c^{n}\right\rangle_{\beta} \equiv \frac{1}{Z(\beta)} \int_{0}^{\infty} c^{n} p^{(\mathrm{F})}(c) e^{-\beta c} d c .
$$

Note that $\langle c\rangle_{\beta}=D$. This is a natural result. As the aggregate demand $D$ rises, workers move to firms with higher productivity. It corresponds to the higher temperature due to the weight factor $e^{-\beta c}$.

(ii) For $T \rightarrow 0(\beta \rightarrow \infty)$,

$$
D \rightarrow 0
$$

This is evident from the fact that in the same limit the integration in Eq.(9) is dominated by $c \simeq 0$ due to the factor $e^{-\beta c}$.

(iii) For $T \rightarrow \infty(\beta \rightarrow 0)$,

$$
D \rightarrow \int_{0}^{\infty} c p^{(\mathrm{F})}(c) d c\left(=\langle c\rangle_{0}\right)
$$

This can be established based on the property (i) because $D=\langle c\rangle_{\beta} \rightarrow\langle c\rangle_{0}$ as $\beta \rightarrow 0$ and $Z(0)=1$.

Let us now study the small $\beta$ (high temperature) properties. One possible approximation for Eq.(9) is obtained by expanding the factor $e^{-\beta c}=1-\beta c+\cdots$ and 
carrying out the $c$-integration in each term. This leads to the following:

$$
\begin{aligned}
Z(\beta) & =\int_{0}^{\infty} p^{(\mathrm{F})}(c)\left(1-\beta c+\frac{1}{2}(\beta c)^{2}+\ldots\right) d c \\
& =1-\langle c\rangle_{0} \beta+\frac{1}{2}\left\langle c^{2}\right\rangle_{0} \beta^{2}+\ldots,
\end{aligned}
$$

where we have used the normalization condition,

$$
\int_{0}^{\infty} p^{(\mathrm{F})}(c) d c=1
$$

The result (33) is, however, valid only for $\mu_{\mathrm{F}}>2$, since $\left\langle c^{2}\right\rangle_{0}$ is infinite for $\mu_{\mathrm{F}} \leq 2$, which fits our observations.

The correct expansion for $1<\mu_{\mathrm{F}}<2$ is done in the following way. We first separate out the first two terms in the expansion of the factor $e^{-\beta c}$;

$$
\begin{aligned}
Z(\beta) & =\int_{0}^{\infty} p^{(\mathrm{F})}(c)\left(1-\beta c+\left(e^{-\beta c}-1+\beta c\right)\right) d c \\
& =1-\langle c\rangle_{0} \beta+Z_{2}(\beta), \\
Z_{2}(\beta) & \equiv \int_{0}^{\infty} p^{(\mathrm{F})}(c) g(c) d c=\int_{0}^{\infty}\left(-\frac{\partial}{\partial c} P_{>}^{(\mathrm{F})}(c)\right) g(c) d c \\
& =\int_{0}^{\infty} P_{>}^{(\mathrm{F})}(c) \frac{\partial g(c)}{\partial c} d c
\end{aligned}
$$

where $g(c)=e^{-\beta c}-1+\beta c$ is a monotonically increasing function of $c$ with

$$
g(0)=g^{\prime}(0)=0 \text {. }
$$

The $c$-integration in Eq.(36) is dominated by the asymptotic region of $c$ for small $\beta$. Therefore, the leading term in $Z_{2}(\beta)$ is evaluated by substituting the asymptotic expression of $p^{(\mathrm{F})}(c)$;

$$
P_{>}^{(\mathrm{F})}(c) \simeq\left(\frac{c}{c_{0}}\right)^{-\mu_{\mathrm{F}}}
$$

into Eq.(36). We thus arrive at the following:

$$
\begin{aligned}
Z_{2}(\beta) & =\int_{0}^{\infty}\left(\frac{c}{c_{0}}\right)^{-\mu_{\mathrm{F}}} \frac{\partial g(c)}{\partial c} d c+\cdots \\
& =\mu_{\mathrm{F}} \Gamma\left(-\mu_{\mathrm{F}}\right)\left(c_{0} \beta\right)^{\mu_{\mathrm{F}}}+\cdots
\end{aligned}
$$

The case $\mu_{\mathrm{F}}=2$ can be obtained by taking the limit $\mu_{\mathrm{F}} \rightarrow 2+$ in the following expansion valid for $2<\mu_{\mathrm{F}}<3$ :

$$
Z(\beta)=1-\langle c\rangle_{0} \beta+\frac{1}{2}\left(\left\langle c^{2}\right\rangle_{0}-\langle c\rangle_{0}^{2}\right) \beta^{2}+\mu_{\mathrm{F}} \Gamma\left(-\mu_{\mathrm{F}}\right)\left(c_{0} \beta\right)^{\mu_{\mathrm{F}}}+\ldots
$$


which can be obtained in the manner similar to the above. The third term is finite for $\mu_{\mathrm{F}}>2$, but diverges as $\mu_{\mathrm{F}} \rightarrow 2+$ as

$$
\left\langle c^{2}\right\rangle_{0} \rightarrow \frac{2 c_{0}^{2}}{\mu_{\mathrm{F}}-2} .
$$

This cancels the divergence of the fourth term in the same limit and the remaining leading term is as follows:

$$
Z(\beta)=1-\langle c\rangle_{0} \beta-\left(c_{0} \beta\right)^{2} \log \left(c_{0} \beta\right)+\ldots
$$

In summary, the partition function behaves as follows:

$$
Z(\beta)= \begin{cases}1-\langle c\rangle_{0} \beta+\frac{1}{2}\left\langle c^{2}\right\rangle_{0} \beta^{2}+\ldots & \text { for } 2<\mu_{\mathrm{F}} \\ 1-\langle c\rangle_{0} \beta-\left(c_{0} \beta\right)^{2} \log \left(c_{0} \beta\right)+\ldots & \text { for } \mu_{\mathrm{F}}=2 ; \\ 1-\langle c\rangle_{0} \beta+\mu_{\mathrm{F}} \Gamma\left(-\mu_{\mathrm{F}}\right)\left(c_{0} \beta\right)^{\mu_{\mathrm{F}}}+\ldots & \text { for } 1<\mu_{\mathrm{F}}<2 .\end{cases}
$$

Substituting the above in Eq.(7), we obtain the following:

$$
D= \begin{cases}\langle c\rangle_{0}-\left(\left\langle c^{2}\right\rangle_{0}-\langle c\rangle_{0}^{2}\right) \beta+\ldots & \text { for } 2<\mu_{\mathrm{F}} \\ \langle c\rangle_{0}+2 c_{0}^{2} \beta \log \left(c_{0} \beta\right)+\ldots & \text { for } \mu_{\mathrm{F}}=2 ; \\ \langle c\rangle_{0}-\mu_{\mathrm{F}}^{2} \Gamma\left(-\mu_{\mathrm{F}}\right) c_{0}^{\mu_{\mathrm{F}}} \beta^{\mu_{\mathrm{F}}-1}+\ldots & \text { for } 1<\mu_{\mathrm{F}}<2 .\end{cases}
$$




\section{References}

Aoki, M. and H. Yoshikawa (2007). Reconstructing Macroeconomics - A Perspective from Statistical Physics and Combinatorial Stochastic Processes, Cambridge University Press.

Aoyama, H., H. Yoshikawa, H. Iyetomi, and Y. Fujiwara (2008). Productivity Dispersion: Fact, Theory and Implications, arXiv:0805.2792, RIETI discussion paper 08-E-035.

Aoyama, H., H. Yoshikawa, H. Iyetomi, and Y. Fujiwara (2009). Labour Productivity Superstatistics, Progress of Theoretical Physics: Supplement, 179, 80.

Ausloos, M. and R. Lambiotte (2006). Brownian Particle Having a Fluctuating Mass, Physical Review E, 73 (1), 11105.

Beck, C. (2005). Superstatistics: Recent Developments and Applications, arXiv:cond-mat/0502306v1.

Beck, C. (2008). Recent Developments in Superstatistics, arXiv:0811.4363v1.

Beck, C. and E. G. D. Cohen (2003). Superstatistics, Physica A, 322, 267.

Credit Guarantee Corporations, www.zenshinhoren.or.jp/file/epamph.pdf.

Credit Risc Database Association, www.crd-office.net/CRD/english/ index.htm.

Ikeda, Y. and W. Souma (2009). International Comparison of Labor Productivity Distribution for Manufacturing and Non-Manufacturing Firms, Progress of Theoretical Physics: Supplement, 179, 93.

Kleiber, C. and Samuel Kotz (2004). Statistical Size Distributions in Economics and Actuarial Sciences, Hoboken, New Jersey: John Wiley and Sons, Inc.

Luczka, J. and B. Zaborek (2004). Brownian Motion: A Case of Temperature Fluctuations, Arxiv preprint cond-mat/0406r08.

Nikkei Media Marketing, Inc. (2008). www.nikkeimm.co.jp/english/ index.html.

Nikkei Net Interactive (2008). http://www.nni.nikkei.co.jp/.

Pareto, Vilfredo (1896). Cours D'Économie Politique, Lausanne: F. Rouge.

Souma, W., Y. Ikeda, H. Iyetomi, and Y. Fujiwara (2009). Distribution of Labour Productivity in Japan over the Period 1996-2006, Economics: The OpenAccess, Open-Assessment E-Journal, Vol. 3, 2009-14.

Yoshikawa, H. (2003). The Role of Demand in Macroeconomics, The Japanese Economic Review, 54, 1. 


\section{conomics}

The Open-Access, Open-Assessment E-Journal

\section{Please note:}

You are most sincerely encouraged to participate in the open assessment of this article. You can do so by either rating the article on a scale from 1 (bad) to 5 (excellent) or by posting your comments.

Please go to:

www.economics-ejournal.org/economics/journalarticles/2009-22

The Editor 